\title{
Comparison of Fault Simulation Over Custom Kernel Module Using Various Techniques
}

\author{
Syeda Huma Jabeen ${ }^{2}$, Zhai Gaoshou², Muhammad Ejaz Sandhu', \\ Naima Noor ${ }^{1}$, Rana Hakeem Ali Mudassar ${ }^{1}$ \\ 'Department of ComputerScience, The Institute of Management Sciences, Lahore, Pakistan. \\ ${ }^{2}$ Beijing Jiaotong University, China. \\ (Received: 19 July 2021 ; Accepted: 08 Sep 2021 ; Issue Published: 12 Sep 2021)
}

\begin{abstract}
To test the behavior of the Linux kernel module, device drivers and file system in a faulty situation, scientists tried to inject faults in different artificial environments. Since the rarity and unpredictability of such events are pretty high, thus the localization and detection of Linux kernel, device drivers, file system modules errors become unfathomable. 'Artificial introduction of some random faults during normal tests' is the only known approach to such mystifying problems. A standard method for performing such experiments is to generate synthetic faults and study the effects. Various fault injection frameworks have been analyzed over the Linux kernel to simulate such detection. The following paper highlights the comparison of different approaches and techniques used for such fault injection to test Linux kernel modules that include simulating low resource conditions and detecting memory leaks. The frameworks chosen to be used in these experiments are; Linux Text Project (LTP), KEDR, Linux Fault-Injection (LFI), and SCSI.
\end{abstract}

KEYWORDS: Device Drivers, Fault simulation, KEDR Framework, Linux, Linux Test Project (LTP), Linux Fault-Injection (LFI), memory leak detection, SCSI

\section{INTRODUCTION}

Linux kernel-based Operating systems (OS) are used widely in the world. More than $90 \%$ of the most powerful supercomputers, based on that. Because the performance of Linux is superior as compared to other operating systems [1,2]. However, Android OS-based mobile devices dominate the international market [3]. There is still an increasing opportunity to use Linux in enterprise systems, where users expect very high reliability [4]. One of the critical components of the Linux kernel is device drivers. However, there are many other essential kernel components, such as networking stacks, file systems, sound, video infrastructures, and subsystems providing virtualization support, hard drives, removable drives, and network adapters. Fault injection (FI) or fault simulation techniques are generally used to minimize or overcome the difficulties by regulating the faults and executing error handling code [5]. Also helps to evaluate the system's tolerance and predict the system failure [6]. Different fault injection tools or techniques are available for Linux fault injection or fault simulation. Still, none of them can inject various kinds of fault scenarios or are not flexible to inject faults as one wants to inject, which is required for systematic assessment of error handling code [4]. Linux kernel module testing by fault simulation is a critical task. It differs from user-space applications; Linux kernel modules might have direct access to some memory areas used by other parts of the Kernel [7]. Kernel modules may communicate at a lower level with the hardware and may have some other capabilities that are hardly available outside the Kernel. Furthermore, it should be noted that any type of kernel module resource allocation will not be freed automatically even when one unloads the module. So, it should be noted that a faulty kernel module is very harmful if it gets out of control [8].

The rest of the paper is structured as follows. In Section II, we describe Categories and Tools of Fault Injection. Then, in Section III, we present our experimental setup. In Section IV, we provide and discuss the results of our experiments and summarize our contributions. In Section $\mathrm{V}$ we propose topics for future work.

\section{OVERVIEW OF VARIOUS TOOLS AND CATEGORIES OF FAULT INJECTION}

In the following section, we explain the different categories and tools of fault injection.

\subsection{Fault Injection Categories}

Fault injections are essential techniques for authenticating the reliability of a system by examining the performance of the devices when a fault happens. Numerous efforts have been made to develop methods for inserting faults into a system prototype. Most of the advanced techniques are divided into five main types, as shown in Figure 1. The significant categories of Fault injection are specified below

\subsubsection{Hardware-Based Fault Injection}

Hardware-based fault injections require expert knowledge and manual testing. However, their results are unquestionable [9]. It's a kind of physical fault injection. In which faults are injected by heavyion radiation, electromagnetic-based interference like voltage or power supply disability, laser [10]. Hardware fault injections can be sub-categorized 
based on their faults and locations, i.e., with contact and without contact [11].

\subsubsection{Software-Based Fault Injection}

Software-based fault injection (SWIFI) alters the contents of memory or registers the occurrence of fault simulation. In Software-based fault injection, different errors or bugs are introduced at a software level that would result in the production of faults in hardware. For speedy coverage, Software-based fault injections depend upon simulations [9]. Software injection can further be categorized on how the Fault is injected:[11].

- Compile-time fault injection

- Runtime fault injection

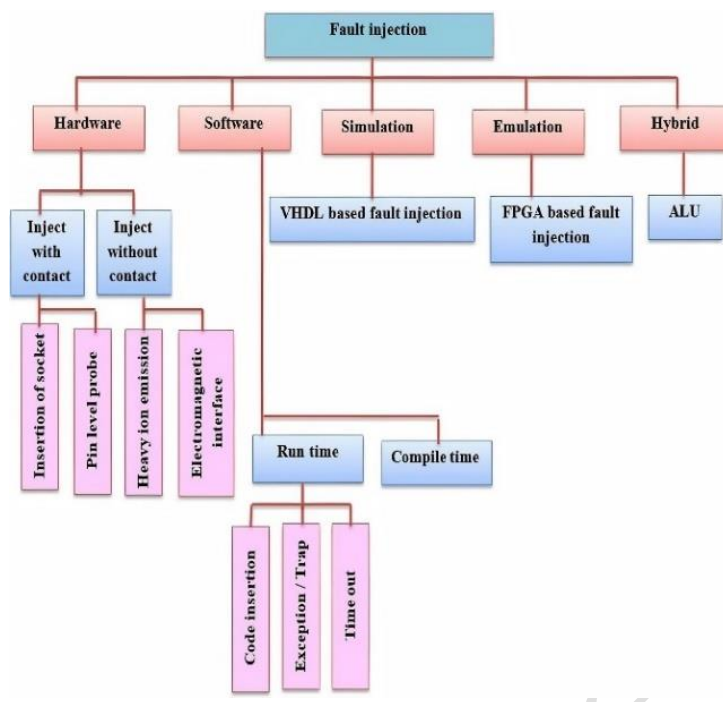

Figure 1: Types of Fault Injection.

\subsubsection{Simulation-Based Fault Injection}

The technique is applied to high-level fault models, for example, to VHDL models. It is beneficial in evaluating the model when it is just in the initial state. It tests the various abstraction levels by utilizing distinct kinds of descriptive languages [10]. Faults propagated during simulation by changing the model's logical values. This approach mainly focuses on injecting Fault primarily on VHDL models and systems [12].

\subsubsection{Emulation Based Fault Injection}

This technique is used to study the actual behavior of the circuit in the environment application considering real-time interactions. Its FPGAs are used for speeding up fault simulation and effective circuit emulation [13]. It can be very timeconsuming [17].

\subsubsection{Hybrid Fault Injection}

This approach is a combination of both softwareimplemented fault injection and hardware. The Hybrid Fault injection technique can be grouped into the following:
- Invasive Techniques: The techniques that leave behind footprints during testing. The problem of sufficiently complex systems, especially time-dependent systems, is that it is usually impossible to remove the footprint of the testing processes from system behavior, independent of fault injections.

- Non-invasive Techniques: These can hide their presence without affecting a system other than the fault injection [15].

\section{METHODOLOGY AND EXPERIMENTAL SETUP}

Several techniques and tools are being used for the Linux kernel module testing; some of the unique tools are reviewed in this paper, as shown in Figure 2. Fault injection tools can be categorized as follows:

- Static Analysis Tools: The tools that only analyze the source code of the module.

- Dynamic Analysis Tools: The tools, which perform runtime, post mortem, or both. The Fault is injected into the circuit, and then the different input vectors are simulated in the circuit to compute faults [16].

It is helpful to use both kinds of analysis, i.e., static and dynamic kernel modules testing [17].

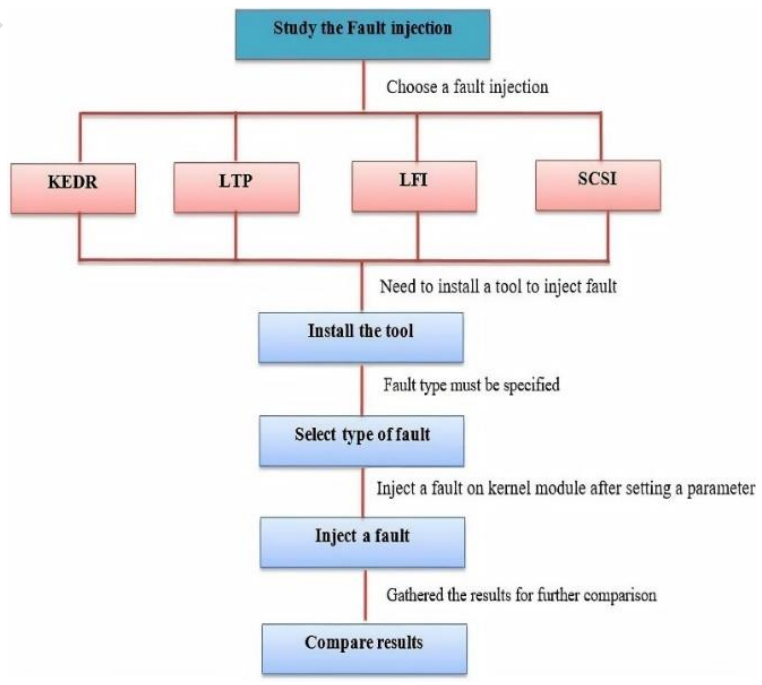

Figure 2: Tools of Fault Injection

In this section, we have conducted experiments by injecting frameworks over the Linux kernel. Most of the frameworks used in these experiments concatenated with kernel fault injection framework (LFI) as provided by Linux version 2.6.20. Each framework has its specialty to fault type and behavior while injecting a fault on Linux kernel.

\subsection{Linux Fault-Injection (LFI) framework}

Starting from version 2.6.20, the Linux kernel has its fault injection called Linux Fault-Injection (LFI) framework to inject various faults into memory and 
block devices. The Linux fault injection framework supports runtime patterns. LFI framework is directed in both ways, i.e., user-space (applicationlevel) and five system-level targets. There is a configuration to activate the Linux FI framework, i.e., a set of files in the debug file-system to divert the Kernel toward a specific point where faults should be injected. This can be done automatically by an application or through a script. According to user interest, fault injection mechanisms can be altered and enhanced for specific points of interest because Linux is free software and open-source. Like Figure 3, the workflow of LFI shows how we have used this framework to insert faults. As of version 4.9.5., the framework can allocate memory in the Linux kernel module mainly in three ways:

- Page allocations errors:

- Slab errors:

- Disk I/O errors:

Various systems are now included in Linux kernel like Kmemleak, Kmemcheck, FI framework, etc. [18].

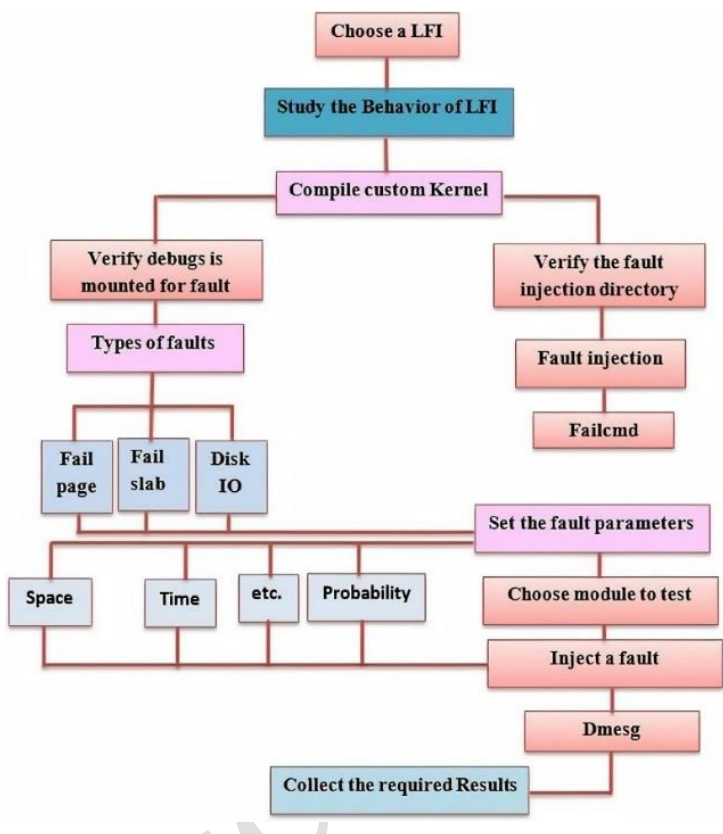

Figure 3: Workflow of LFI

\subsection{Experiment on Linux Fault-Injection (LFI)}

To implement the fault injection by using LFI, (i) VMware station 10.0 is installed on our machine, and a virtual machine is built, and (ii) Ubuntu 15.04 with Linux-header-3.19.0-15-generic is installed on that virtual machine. Then source codes of (iii) LFI are downloaded, compiled and installed on the virtual machine.

\subsubsection{Compilation of custom kernel in Linux Fault-Injection (LFI)}

The Compilation of the custom kernel consists of two ways:

- The traditional way to compile the Kernel.
- The latest and easy way to compile the Kernel.

3.2.2. Fault Injection Capabilities and Configuration of Capabilities Behavior in Linux Fault-Injection (LFI)

After reviewing the behavior of LFI, it was acknowledged that the Linux Fault Injection Framework is required for Debugfs entries to satisfy the fault injection capabilities. There are mainly two ways to make Kernel satisfied for fault injection capabilities, as shown in Table 1.[20]:

- Boot time configuration of fault capabilities.

- Runtime configuration of fault injection.

Table 1. Fault Injection Behavior

\begin{tabular}{|l|l|l|}
\hline $\begin{array}{l}\text { Available Fault } \\
\text { Injection } \\
\text { Capabilities }\end{array}$ & $\begin{array}{l}\text { Boot Time Configuration } \\
\text { setup fault attr(attr, str); }\end{array}$ & $\begin{array}{l}\text { Run-Time Configuration } \\
\text { Debugfs Entries }\end{array}$ \\
\cline { 1 - 2 } Failslab & o Failslab & $\begin{array}{l}\text { Compile a Custom Kernel As } \\
\text { per capabilities of Fault } \\
\text { Injection }\end{array}$ \\
\cline { 1 - 2 } Fail page alloc & o Fail page alloc $=$ & \\
\cline { 1 - 2 } Fail futex & o Fail futex $=$ & \\
\cline { 1 - 2 } Fail to make a & o, Fail to make request $=$ & \\
request & o Fail mmc request $=$ & \\
\cline { 1 - 2 } & &
\end{tabular}

3.2.3. Selection of Faults and Parameters in Linux Fault-Injection (LFI)

After successfully compilating the custom kernel, a fault can be selected to inject by utilizing different types of faults in LFI, i.e. Fail- Page, FailSlab and DiskI/O, as shown in Table 2.

Table 2. Types of Faults.

\begin{tabular}{|l|l|l|l|l|}
\hline \multirow{2}{*}{$\begin{array}{l}\text { Parameter of Faults } \\
\text { in/sys/kernel/debug/ }\end{array}$} & \multicolumn{5}{|c|}{$\begin{array}{c}\text { Types of } \\
\text { Faults }\end{array}$} \\
\cline { 2 - 5 } & $\begin{array}{l}\text { Fail_ } \\
\text { Slab }\end{array}$ & $\begin{array}{l}\text { Fail page } \\
\text { alloc }\end{array}$ & $\begin{array}{l}\text { Fail } \\
\text { make_ } \\
\text { request }\end{array}$ & $\begin{array}{l}\text { Fail io_ } \\
\text { time out }\end{array}$ \\
\hline Verbose & 3 & 3 & 3 & 3 \\
\hline Times & 3 & 3 & 3 & 3 \\
\hline Task-filter & 3 & 3 & 3 & 3 \\
\hline Stacktracef/liter & 3 & 3 & 3 & 3 \\
\hline Space & 3 & 3 & 3 & 3 \\
\hline Require-start & 3 & 3 & 3 & 3 \\
\hline Require-end & 3 & 3 & 3 & 3 \\
\hline Probability & 3 & 3 & 3 & 3 \\
\hline Interval & 3 & 3 & 3 & 3 \\
\hline
\end{tabular}




\subsubsection{Injection of Fault on Module and Tests in LFI}

The Linux Fault Injection Framework has a shell script of fault injection with the name failcmd inside the fault injection directory. An individual may inject a fault on a Single module by providing a module parameter. In addition, it can be injected on Run tests. To inject a fault, fault type may be defined at run time by using a command given below:

\# env FAILCMD TYPE=fail page alloc

./tools/testing/fault-injection/failcmd.sh times $=100-$ make - C tools/testing/selftests/run tests

In this command, parameters are used 100 times, fault type is selected as a Fail - page, and applied to run tests. But if the fault type is not specified, by default, it will Failslab.

\subsection{SCSI Fault Injection tool framework}

The SCSI fault injector is capable of fault injections in a realistic pattern, and it is comprised of a set of various test programs to cover different fault conditions. It is implied as a set of System Tap scripts. SystemTap is mainly used to track information when an I/O request is passed between layers within a kernel [4]. The SCSI fault injection works in two steps.

- It identifies the target SCSI command by matching user-specified.

- Inject Fault in the processing of the target SCSI command.

\subsubsection{Testing Error Handler Using SCSI Fault Injection}

As Figure 4, the Workflow of SCSI illustrates how the SCSI framework is used for fault injection. To get a fruitful result, it is required to have a set of test programs during tests systemic testing for error handling source code by using fault injections for any target kernel component. This set of test programs makes sure either that the Fault is occurring or not while the target kernel component is being handled appropriately. For specific test programs, it is compulsory to have a target kernel component in a preferred state. The specific (desired) Fault is injected in the desired place where it will trigger by test programs, and then the result is tested whether it is correctly injected or not. For such goals, the Tool provides a platform to specify the kind of Fault, which will be injected, and then finally, it will cause injection.

\subsubsection{Specifying the Type of SCSI Fault}

The SCSI HDD fault patterns are classified to define a specific fault pattern generated by the fault injector.SCSI fault can be classified into two patterns. First, "Response of SCSI device with an error pattern", meaning when the drive returns an error condition, it is returned to the OS. For example, it can be caused by a media error. Second, "The unresponsive device pattern", in this case, the drive does not return any error to the OS 15, resulting in a timeout. For example, it can be caused by SCSI cable faults. Instead of that, HDD hardware faults can be classified into (see Table 3):

1. Temporary Fault: It can be generated by an accidental way and recoverable HDD fault.

2. Permanent Fault: A severe HDD fault can cause it.

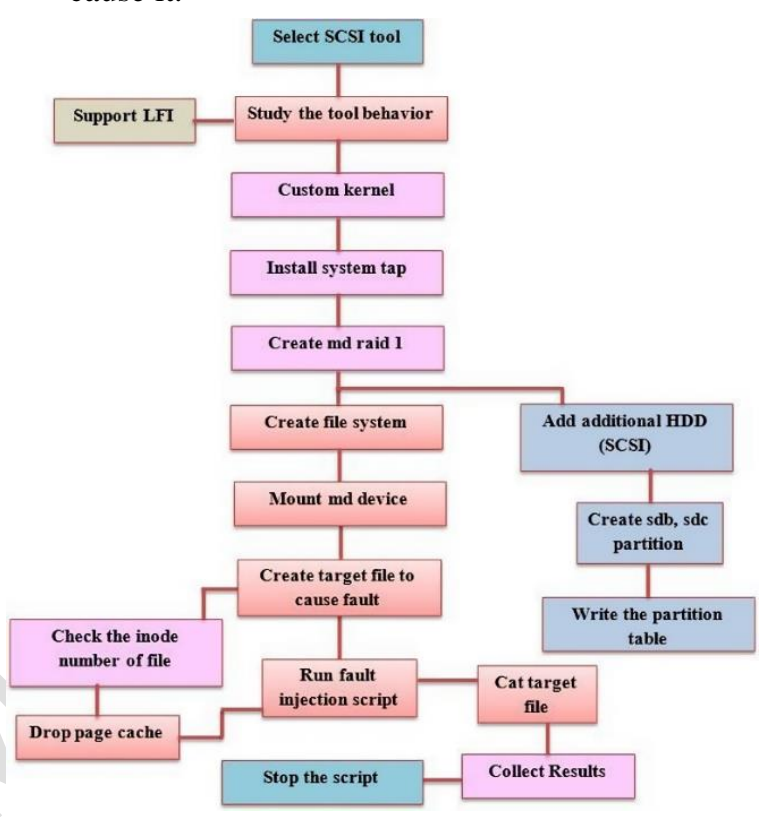

Figure 4: Workflow of SCSI

\subsubsection{Experiment on SCSI}

To inject a fault with a single disk once the target command is found, the SCSI fault injector modifies the target. System Tap provides a platform for the SCSI fault injector to insert a hook inside the Kernel that dynamically also alters the value of variables [4]. While using SystemTap, a false is created from a SCSI device if this reports an error in OS [13]. SCSI tool faults patterns and triggers conditions are obtained from a test program as an argument. Figure 4. Illustrates a Workflow of SCSI. For example, RAID drivers software were tested by injecting different SCSI faults to RAID drivers and to check if the software RAID driver and SCSI middle layer error handling code work properly. 
Table 3. Types of faults offered by SCSI

\begin{tabular}{|l|l|}
\hline Script Name & Fault type description \\
\hline disk,, err.stp & Permanently read error simulation \\
\hline disk, wrr.stp & Permanently read/write error simulation \\
\hline sector,err.stp & $\begin{array}{l}\text { Read error correctable by writing } \\
\text { simulation }\end{array}$ \\
\hline temporany,err.stp & Temporary read error simulation \\
\hline temporary, $6 r$ stp & Temporary write error simulation \\
\hline imeout.stp & $\begin{array}{l}\text { Temporary no response to reading access } \\
\text { simulation }\end{array}$ \\
\hline imeout.stp & $\begin{array}{l}\text { Temporary no response to write } \\
\text { access simulation }\end{array}$ \\
\hline nwimeout.stp & $\begin{array}{l}\text { Permanent no response on both read-write } \\
\text { access simulation }\end{array}$ \\
\hline
\end{tabular}

\subsubsection{Testing Procedure}

The following procedure needs to be evaluated.

- Installing the OS on one of the SCSI disks.

- Injection of various patterns of HDD fault.

- Check the results.

Make custom kernel to support Fault Injection with SCSI

The SCSI fault injector frame is flexible in behaviour, i.e., easy to inject by making simple changing in the Kernel because of its SystemTap scripts [4]. Virtual configurations for the Kernel are given below:

\section{General setup}

a. [*] Kprobes

b. [*] Kernel - >user space relay support (formerly relayfs)

c. [*] Enable loadable modules

\section{Kernel hacking}

a. [*] Kernel debugging

b. [*] Compile the Kernel with debug info

\section{Redundant Array of Independent Disks - RAID}

Disk I/O performance can be improved by creating a single logical unit of multiple hard disks. RAID is a storage technology that mainly focuses on combining multiple disks to create one disk over which Fault can be injected to improve its functionality.

* Raid 1- Mirroring RAID1 array software setup (also recognized as a "mirroring" array), in which the array forms of data are written over two disks. Although it also can generate a RAID-1 on a single hard disk by making a partition over it. It has a drawback with it; failure may occur if that single hard disk faces with Fail.

* MDADM - multiple disks-admin on Linux Mdadm is mainly a tool that helps to generate a RAID of different levels. This tool provides the functionality to manage, assemble, create, and monitor a RAID. It is available by defaults on
LINUX distros such as CentOS, RHEL, Fedora or Arch Linux platforms.

* Creation of RAID device To create RAID 1, firstly, it verifies the number of disks available under the Ubuntu server, using a (fdisk -lu) list of all the available disks. It has found only a single disk sda (system disk).

* Hard Disk Partition While the hard disk has been added successfully; it still has not been mounted on virtual systems. You need to do a hard disk partition and use it. Check the current hard drives and their corresponding partitions, open Terminal and type command: Sudo fdisk - lu . The terminal will show the two prompts given below:

Disk /dev/sdb doesn't contain a valid partition table

Disk /dev/sdc doesn't contain a valid partition table

* Create a sdb system disk After reboot, verify the above functionality has been done successfully. It must add a partition table for $\mathrm{sdb}$, format and automatically mounted in a virtual system. Sdb is created by using a given command:

\section{"fdisk/dev/sdb"}

* Create a sdc system disk The partition table is open by using a given command:

\section{"fdisk/dev/sdc"}

\subsubsection{Install SCSI fault injector source and} mdadm

Download, SCSI tool source forms the GitHub respiratory, extract it on desires place. To install the mdadm command below is used:

\section{Sudo apt-get install mdadm}

\subsubsection{Create md RAID1 array}

To create an md RAID1 array, mdadm is used with multiple options.

$$
\begin{aligned}
& \text { mdadm -C /dev/mdo -l1 -n2 -f } \\
& / \text { dev/sd[bc]1 }
\end{aligned}
$$

Status verification of RAID arrays is done by using the command below

\section{cat /proc/mdstat}

\subsubsection{Injection of Fault on RAID1/dev/md0}

Injecting a fault on RAID1 using a SCSI tool needs to follow the steps below:

1. Create a filesystem After creating RAID1 success, it is time to create a file system for md0. The command below is used to create a file-system $\mathrm{mkfs}-\mathrm{t}$ ext $3 / \mathrm{dev} / \mathrm{md} 0$

2. Mount the md device After successfully creating the file system, it must mount md0 to the desired place. The command given below is used to mount /dev/md0 successfully. 
mount -t ext3 /dev/md0/home/SCSI/Desktop/

SCSI-fault-injection-test-tool-1.0.1

3. Create a target file to cause a fault The file created to inject a fault might be cached in memory, but access to the target device wants to be made to inject a fault. For being sure, must drop all pages by using a command:

"echo $1>/$ proc/sys/VM/drop caches"

Check the inode number of the file by command:

"Is -hil"

It created two prompts as a result of "ls - hil":

Total 28K

11 drwx 2 root $16 \mathrm{~K}$ 2007-12-21 $18: 22$ lost+found

12 -rw-r-r-1 root21 2007-12-21 19:39 test.txt

These prompts showed that:

"test. the text has inode number 12 in the md0 device."

\subsubsection{Install SystemTap}

SystemTap is a crucial feature for the SCSI injector, so ensure whether the platform is installed; if it is not installed, install it by the apt-get SystemTap command. SytemTap takes almost 5-10 minutes, depending upon the processor.

\subsection{LTP Fault Injection tool framework}

Linux Test Project (LTP) is an open-source project, which brings test suites to the open-source developers or the users that authenticate the steadfastness, strength, and constancy of Linux.

The Linux operating system has various aspects that can be tested with the LTP test suite because it contains automatic and semi-automatic tests.

The workflow of LTP is described in Figure 5, which shows how we have used this framework to insert faults.

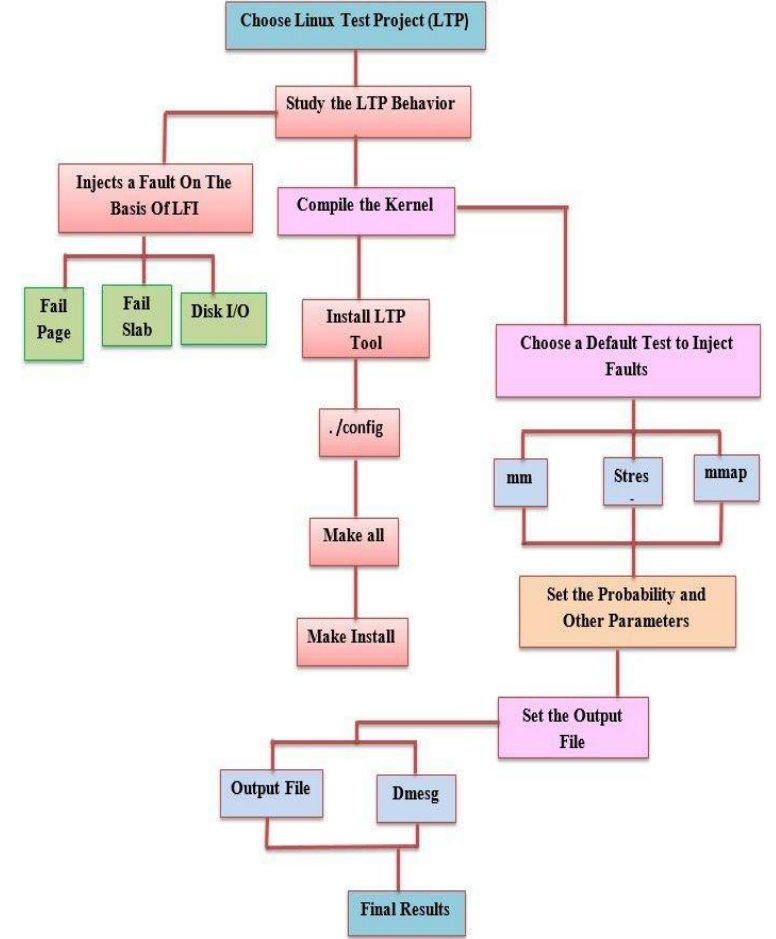

Figure 5. Workflow of LTP

\subsection{Experiments on Linux Test Project (LTP)}

To implement the fault injection by using Linux Test Project (LTP), (i) VMware station 10.0 was already installed on our machine, and a virtual machine is built, and (ii) Ubuntu 15.04 with Linux-header3.19.0-15-generic is installed on that virtual machine. Then source codes of (iii) LTP version 1.1 are downloaded, compiled and installed on the virtual machine. In LTP, Faults can be applied under two conditions:

- $\quad$ Stable Kernel

- Fault Injected kernel (LFI)

3.4.1. Compile a custom kernel for Linux Test Project (LTP)

Mainly LTP is needed to compile a kernel to work with LTP to injection fault with the support of LFI.

\subsubsection{Installation of LTP by Quick Start}

To install the LTP quick start has been chosen, which will reduce the overall installation time and give a fast install.

Table 4. Required Kernel Configuration (KC) for LTP

\begin{tabular}{|l|l|l|l|}
\hline Required KC & Set KC & Required KC & Set KC \\
\hline Fault injection & Y & Fail to make a request & Y \\
\hline Debug kemel & Y & Block & Y \\
\hline Failslab & Y & Fail io timeout & Y \\
\hline Fail page alloc & Y & Fault injection debug fs & Y \\
\hline
\end{tabular}




\subsubsection{Linux Test Project (LTP) supports Linux Fault-Injection (LFI)}

LTP is joined with LFI to inject a fault. Table 4 shows the kernel configuration for LTP.

\subsubsection{Test cases in Linux Test Project (LTP)}

LTP mainly provides various test cases that can be used to verify the Kernel. It provides the network test, disk I/O test, ID check and other kernel code tests under the Runltp directory.

\subsubsection{Runltp Test-cases for fault injection}

Runltp test cases have a script to run an actual test. The test script can be selected manually depending upon the requirements. The script is called runalltest. sh but it does not mean that all tests in the LTP will run at once.

\subsubsection{Injection of Fault on default Test in Linux Test Project (LTP)}

We have chosen a default test (Run test) to inject a fault. However, we need to run a script. Runltp with root privilege.

\subsubsection{A shell script and parameters to inject Fault}

There are mainly two parameters, Loop and Probability, supports for LTP Test suites for fault injection over tests. LTP test shell script is made by Perl; LTP command will be working as input, which will create a final file on the way given below:[12]

\section{<loop1> <binary name is tested > \\ $<$ loop2><insert kernel faults.sh to test binary name $>$}

$<$ loop3> <label passes from loop3 to loop (n1)>

<loopn under kernel fault>-<restore kernel faults default.sh to test binary name $>$

\subsubsection{Injection of faults over choose Test}

\section{Test Case 1}

We have selected the memory management (mm) test as the first test to run under the faulty Kernel; the command is given below:

$$
\begin{aligned}
& \# \text {./runltp -f mm -F 6,15 -o ltp _with fault } \\
& \text { injection.out } \\
& \text { Loop } \rightarrow 6 \\
& \text { Probability } \rightarrow 15
\end{aligned}
$$

Test Case 2

We selected mmapstress as a second test to run under a faulty kernel, a command is given below:

\# ./runltp -f mmapstress -F 8,40 -o ltp with fault injection stress test.out

$$
\begin{aligned}
& \text { Loop } \rightarrow 8 \\
& \text { Probability } \rightarrow 40
\end{aligned}
$$

\section{Test Case 3}

We choose dma thread diotest as a third test to run under a faulty kernel; the command is given below:

$$
\begin{aligned}
& \text { \# ./runltp -f dma thread diotest }-F \text { 6,60 -o } \\
& \text { ltp with fault injection dma thread dio } \\
& \text { test.out } \\
& \text { Loop } \rightarrow 6 \\
& \text { Probability } \rightarrow 60
\end{aligned}
$$

\subsection{KEDR Framework}

We have already worked with this Tool and have published our work. We have used KEDR for the device's read and write operations and memory allocation to inject faults into the related custom kernel module. We found the KEDR framework is efficient and helpful in injecting and simulating a fault [19].

\section{RESULTS}

After successfully injecting a fault by different tools, it is observed which Tool is better in what way. The rating level is selected from 0 - 5 numbers, 0 being the lowest and five being the highest.

Table 5 illustrates the comparison made based on Feature, Support and, Ease. LTP is consistently higher than others except for feature and design, where KEDR is the highest. However, overall, LTP is better. LFI is slower in all aspects, indicating that it is the slowest Tool. On the other hand, SCSI is moderate in all aspects.

Table 5. Comparison made on Feature, Support Ease

\begin{tabular}{|c|c|c|c|c|}
\hline Tools & Feature & Support & Ease & Design \\
\hline KEDR & 5 & 4 & 4 & 5 \\
\hline SCSI & 3.5 & 3 & 3.7 & 2 \\
\hline LFI & 2.5 & 4 & 2 & 2.5 \\
\hline LTP & 4.5 & 5 & 4.5 & 5 \\
\hline
\end{tabular}

A better fault injector tool is the one which supports as many as possible faults injected into it. Kma-lloc, Fail-slab, DiskI/O, Raid and, Char Device were injected in LFI, KEDR and, SCSI to check which tools support which Fault to inject. Table 6 illustrates LFI supported three faults. At the same time, we were succeeded to inject two faults in KEDR and SCSI. Only LFI supports maximum injectors.

Table 6. Fault Based Comparison

\begin{tabular}{|c|c|c|c|c|c|}
\hline Tools & Kma-lloc & Fail-slab & DiskI/0 & Raid & $\begin{array}{l}\text { Char } \\
\text { Device }\end{array}$ \\
\hline LFI & Yes & Yes & Yes & No & No \\
\hline KEDR & Yes & No & No & No & Yes \\
\hline SCSI & No & No & Yes & Yes & No \\
\hline
\end{tabular}


Table 7 illustrates the comparison made based on the KEDR-sample-target module. The KEDR exemplary module is verified under different conditions to check whether the KEDR-sample module is supported under different conditions or not. The first task was under normal conditions (no simulator); in this condition, the KEDR-sample target loaded successfully with its device driver but failed to inject the Fault. After that, it was verified in LFI; the KEDR-sample target loaded successfully with its device driver in this condition. However, it didn't support the injection of Fault. The condition KEDR-sample target loaded successfully along with its device driver in KEDR, with no error.

Table 7. Compare Kedr-sample-target under KEDR, NORMAL and LFI.

\begin{tabular}{|c|l|l|l|l|}
\hline Conditions & $\begin{array}{l}\text { Successfully } \\
\text { load module }\end{array}$ & $\begin{array}{l}\text { Load the } \\
\text { char } \\
\text { Devices } \\
\text { cfake0, } \\
\text { cfakel) }\end{array}$ & $\begin{array}{l}\text { Verify the } \\
\text { devices }\end{array}$ & $\begin{array}{l}\text { Successfully } \\
\text { injected Fault }\end{array}$ \\
\hline Normal & Yes & Yes & Yes & No \\
\hline LFI & Yes & Yes & Yes & $\begin{array}{l}\text { Do not support } \\
\text { module }\end{array}$ \\
\hline KEDR & Yes & Yes & Yes & Yes \\
\hline
\end{tabular}

Every test on each Tool is needed for a different space level, as shown in Figure 6. LTP needs maximum space between them while KEDR needs the least space that is $20 \mathrm{~GB}$. SCSI and LFI space depend upon custom kernel configuration, and it is clear that the custom kernel needs more significant than 2 GB so, SCSI needs an additional space of 15 $\mathrm{G}(25 \mathrm{~GB}+15 \mathrm{~GB}=40)$ while LFI requires more significant than $30 \mathrm{~GB}(25 \mathrm{~GB}+5 \mathrm{~GB}=30 \mathrm{~GB})$.

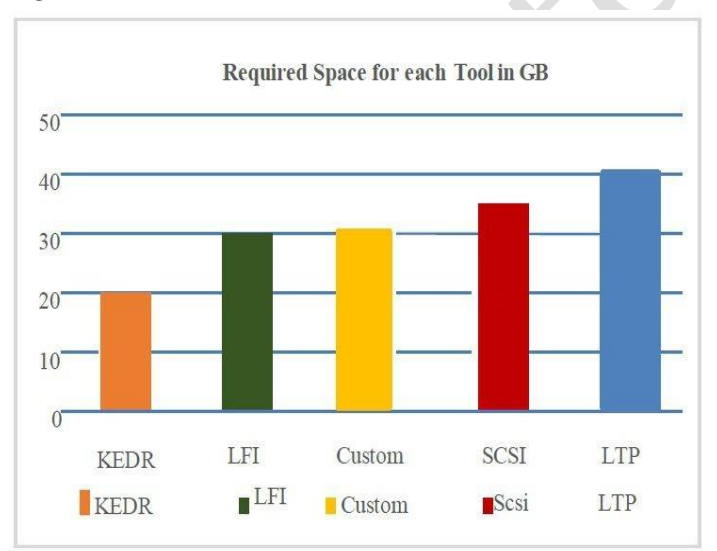

Figure 6: Comparison of Time Requirements.

The time required for each Tool depends upon the kind of test being tested at that time. As shown in Figure 7, a significant amount of time is required for LTP, some test of under the fault kernel takes about $20 \mathrm{hrs}$ and some test just finished under $1 \mathrm{hr}$. The least time is required for the KEDR fault injector. SCSI required time is also dependable over the test $1 / 2-3 \mathrm{hr}$. custom kernel time is processor and configuration dependent if the processor is fast and configuration are less in number than time can be 5 hrs. Still, otherwise, it may lead to $\geq 7 \mathrm{hrs}$.

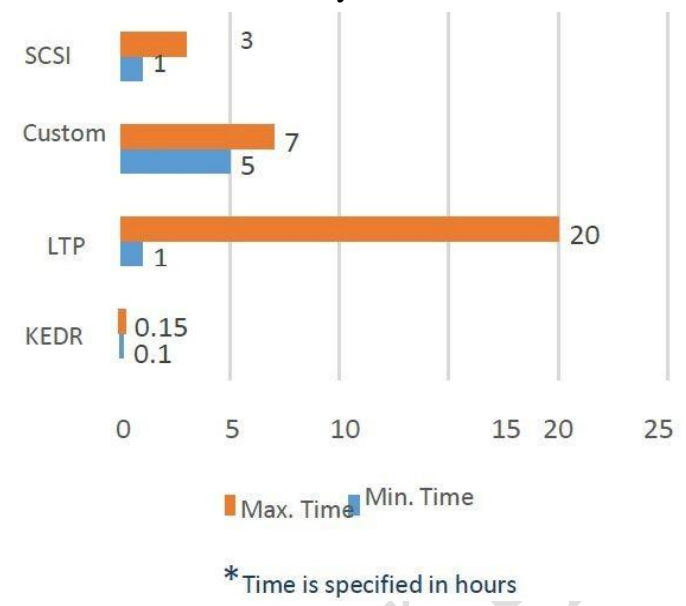

Figure 7: Time Required for Each Tool.

\section{CONCLUSION}

For LTP (Linux Testing Project), we performed three tests under the faulty Kernel; probability percentage and numbers of loops varied each time. KEDR tool was used to study fault simulation and detection of memory leaks overbuilt in the module (Kedr-sample-target) and custom kernel module (Example LKM). SCSI tool was used to inject Fault under the RAID1. The mdadm approach was used to create RAID1.

Comparative experiments have been done based on i) Feature, Support, and Ease ii) Fault support iii) KEDR-sample-target over different conditions iv) Space requirements v) Time requirements of each Tool and reviewing various tools for runtime analysis of Linux kernel modules testing by fault simulation brought us to the conclusion that there are no definitive solutions to this problem.

\section{FUTURE WORK}

Fault can be injected at a specific kernel module or device driver, while a different fault can be specified each time. However, there is still a need for more research in this area of study because the existing Fault Injection Frameworks and tools can not satisfy the requirement entirely and correctly. To improve and make it more ideal, the goal is to test some real devices such as pcnet32, speaker, camera, etc. This experiment will further help to design a fault injection tools better than the existing one used in this experiments, with aspects to time (designed Tool take less time to evaluate an experiment compared to existing), space (take less space to occupy will installation and injecting a fault over a target), support (must be Linux supportive more than others so it will be easy to set up a platform), design and feature ( must be versatile in feature and design that supports for fault injection over the kernel source codes, actual device drivers and custom kernel indeed) and efficient and comfortable 
( must be easy to operate and efficient in behaviour that provides the precise result in a short interval of time).

\section{REFERENCES}

[1] N. S. Sulaiman and A. S. H. A. Raffi, "Comparison of Operating System Performance Between Windows 10 and Linux Mint"," IJSET, vol. 2, no. 1, pp. 92-102, 2021.

[2] Q. Zhu, "The application perspective of mutatoin testing," Delft University of Technology, Netherlands, 2020.

[3] Tsyvarev and A. Khoroshilov, "Using fault injection for testing Linux kernel components," Proceedings of the Institute for System Programming 2015, vol. 27, no. 5, pp. 157-174.

[4] K. Tanaka, M. Hamaguchi, T. Sato, and K. Tatsukawa, "SCCI fault injection test,"Vol 4, issue 3, pp. 205-214, 2008.

[5] Khosrowjerdi, Hojat, Karl Meinke, and Andreas Rasmusson. "Virtualized-fault injection testing: A machine learning approach." In 2018 IEEE 11th International Conference on Software Testing, Verification and Validation (ICST), pp. 297-308. IEEE, 2018.

[6] S. A. Alazawi and M. N. Al-Salam, "FIBROSS: fault injection model for bug reports in open-source software," Indonesian Journal of Electrical Engineering and Computer Science, vol. 20, no. 1, pp. 465-474, 2020.

[7] Khoroshilov, V. Kuliamin, and A. Petrenko, "Verification of Operating System Components," Системная информатика, vol. 10, pp. 11-22, 2017.

[8] Rubanov, Vladimir V., and Eugene A. Shatokhin. "Runtime verification of linux kernel modules based on call interception." 2011 fourth IEEE international conference on software testing, verification and validation. IEEE, 2011.

[9] T. Given-Wilson, N. Jafri, and A. Legay, "Combined software and hardware fault injection vulnerability detection," Innovations Syst Softw Eng, vol. 16, pp. 101-120, 2020.

[10] P. Koppenstein, "Fast user-mode fault injection with FAIL,"Tu-dortmund.de. [Online]. Available: https://ess.cs.tudortmund.de/Teaching/Theses/2020/MA_Kop penstein_2020.pdf. [Accessed: 23-Sep-2021].
[11] Koren and C. Mani Krishna, Fault-Tolerant Systems. Morgan Kaufmann, vol 5, issue 3, pp $1-14,2014$

[12] Gil, J. Gracia, J. C. Baraza, and P. J. Gil, "Study, comparison and application of different VHDL-based fault injection techniques for the experimental validation of a fault-tolerant system," Microelectronics, vol. 34, no. 1, pp. 41-51, 2003.

[13] L. Antoni, R. Leveugle, and M. Feher, "Using runtime reconfiguration for fault injection in hardware prototypes," in 17th IEEE International Symposium on Defect and Fault Tolerance in VLSI Systems, 2002. DFT 2002. Proceedings, 2003.

[14] J. Roux et al., "Cross layer fault simulations for analyzing the robustness of RTL designs in airborne systems," in 2020 23rd International Symposium on Design and Diagnostics of Electronic Circuits \& Systems (DDECS), 2020.

[15] Yu, Yangyang, and Barry W. Johnson. "Fault Injection Techniques." In Fault Injection Techniques and Tools for Embedded Systems Reliability Evaluation, pp. 7-39. Springer, Boston, MA, 2003.

[16] M. Eslami, B. Ghavami, M. Raji, and A. Mahani, "A survey on fault injection methods of digital integrated circuits," Integration, vol. 71, pp. 154-163, 2020.

[17] Gerlits, V. V. Kuliamin, A. V. Maksimov, A. K. Petrenko, A. V. Khoroshilov, and A. V. Tsyvarev, "Testing of operating systems," Proc. Inst. Syst. Program. RAS, vol. 26, no. 1, pp. 73-108, 2014.

[18] Apte, Varsha, and Bhavin Doshi. "Powerperfcenter: A power and performance prediction tool for multi-tier applications." Proceedings of the 5th ACM/SPEC international conference on Performance engineering. 2014.

[19] Jabeen, Syeda Huma, Gaoshou Zhai, and Ruixia Zhai "Fault Simulation and Memory Leak Detection over Custom Kernel Module by Using KEDR,”2017, pp. 244-248 2017.

[20] Naughton, Thomas, et al. "Fault injection framework for system resilience evaluation: fake faults for finding future failures." Proceedings of the 2009 workshop on Resiliency in high performance,pp 22-28, 2009. 


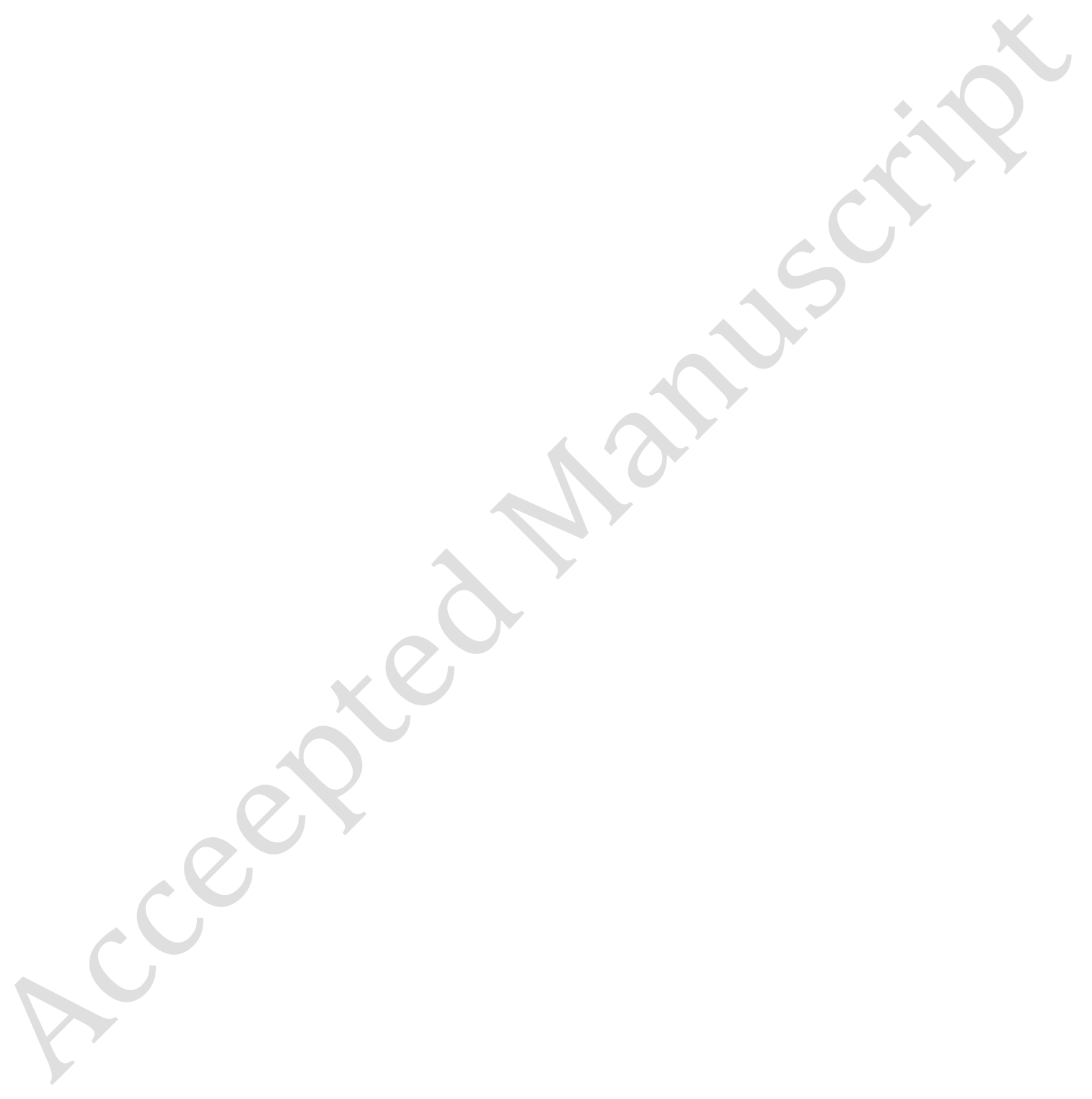

